\title{
Energy Efficiency Analysis between Green computing and Cloud Computing
}

\author{
J Kavitha, Rokesh Kumar Yarava
}

\begin{abstract}
Usually cloud computing is based under effective data computing but green cloud computing focus on energy efficiency of device and computing mainly based on computing architecture. Here cloud computing is also known as green computing due its better effective energy. The purpose of green computing is to decrease the usage of harmful resources, maximizing energy efficiency throughout the living span of the good and also to promote the recyclability or bio degradability of useless goods plus misuse materials of the factory. In order to simulate the hardware by utilizing software, green computing plays a major role for various technologies like virtualization, green data center, cloud and grid computing and power optimization. Here computer resources are effectively utilized by replacing the data center standalone server system by artificial server so as to run the software by less number for large computers called as virtualized. Finally this paper is fulfilled by the advancement of recognized energy efficient computing.

Keywords: Cloud Computing, Green data center, Power optimization, Energy efficiency.
\end{abstract}

\section{INTRODUCTION}

Green computing and green technology are referred as ecofriendly by the usage of computer technology as well as technology related to other resources. The execution of energy effective central processing unit plus its peripherals and servers are included in green computing.

The main purpose of green technology is to reduce consumption of resources and also to improve the electronic waste (e-waste) disposal.

Green computing is huge in rising area that is intended for reduction energy that becomes the top priority in all segments of IT market and it is also needed for energy effectiveness that became a serious issue in designing of effective presented computing.
Revised Manuscript Received on October 25, 2019.

J Kavitha, Assistant Professor, Dept. of CSE, Malla Reddy Engineering college (Autonomous), Hyderabad, India.

Rokesh Kumar yarava, Assistant Professor, Dept. of CSE, Malla Reddy Engineering college (Autonomous), Hyderabad, India.

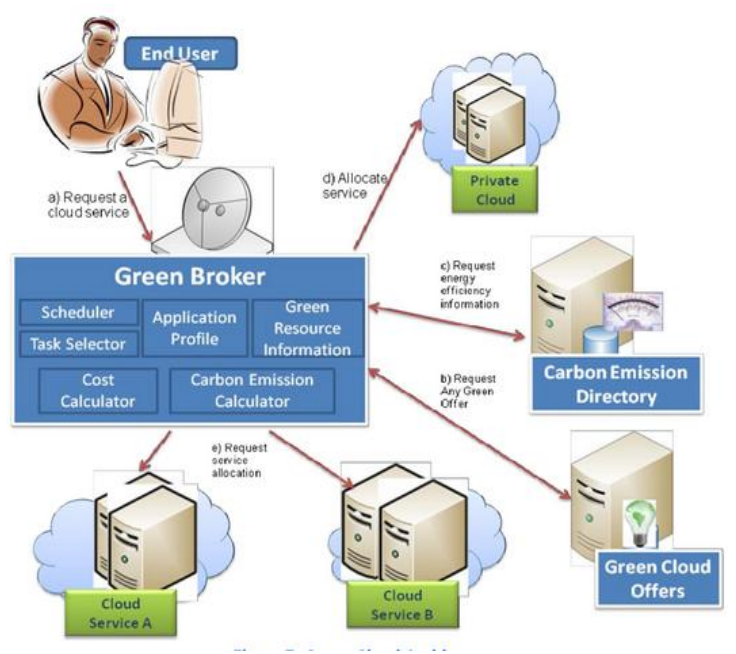

Fig 1 cloud computing for green computing

Those growing accessibility about high speed internet plus corporate IP associations may be empowering the delivery novel network-based services. Same time Internet-based mail administrations have been operating to a significant number years, management contributions bring stretched with fit in network-based ability plus network-based registering. These new administrations would be advertised both will corporate also distinct limit clients. Administrations about this sort need been generically known as cloud registering services. Those cloud registering administration model includes those provision, by a administration provider, for vast pools for high-octane registering assets and high-capacity capacity units that would imparted around wind clients as needed. There are a lot of people cloud administration models, in any case generally, conclusion clients subscribing of the administration need their information facilitated Toward the service, Also need registering assets allocated on interest from those pool. That administrative provider's advertising might additionally stretch out to the product requisitions obliged toward the end client [2]. With make successful, those cloud administration model likewise obliges a high-sounding system on give acceptable connection among those last client plus the administration provider infrastructure.

Cloud registering possibly offers a general budgetary benefit, in that end clients stake a large, centrally figured out how pool of stockpiling furthermore registering resources, as opposed owning and overseeing their systems. Frequently utilizing existing information centers likewise a basis, cloud administration suppliers put

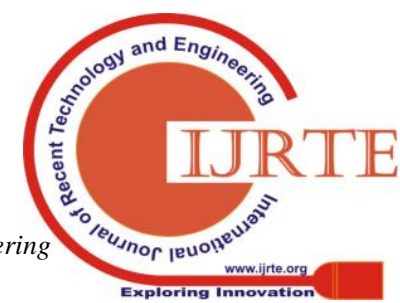




\section{Energy Efficiency Analysis between Green computing and Cloud Computing}

resources into those important foundation and management.

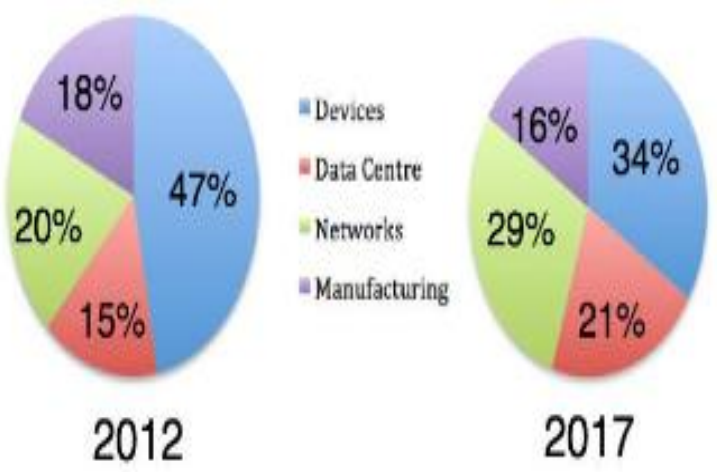

Figure.2. Electricity consumption by main components in ICT Sector

Figure 2, graphically depicts those examination about subside Corcoran and Anders Andrae for "Emerging patterns done power utilization for customer ICT" [15]. Assessing data, field records, Furthermore former researches, the writers have guessed that by the quite a while 2017, those power necessities of the cloud information Centers will bring arrived at an amazing $21 \%$. This will be a disturbing conclusion, which might help gigantic sums for carbon dioxide emanation over our officially hampered surroundings. Various requisitions would run by information centers that fill in under cloud registering. Portion information will be transformed inside seconds, whereas, expansive information nourishing looking into imparted fittings stage takes more occasion when methodology will. Those cloud registering on-demand office countenances tests at different information will be with make processed, one by one alternately simultaneously. Asset Furthermore chance allotment is must with keep away from delay Also generation about wrong comes about [9].

Information centers are unavoidable; they are necessary for information storing, accessing, preparing and other administrations. The rate from claiming Vitality utilization may be alarmingly high, and the significant downside on this utilization is that, just a little portion for this vitality will be really used to generate a yield. The information centers need aid heavy spender on keep up, the extra foundation such as coolers, will administer those temperature about vast number from claiming servers inside an information Centre, includes of the expense and in addition the carbon foot shaped impression. Green cloud registering aides with those convoluted procedure for accomplishing decrease to Vitality utilization amidst proficient utilization of base.

\section{GREEN COMPUTING TECHNIQUE MEANT FOR ENERGY EFFICIENCY.}

Comprehension the routes done which control utilization effects those "greenness" of At whatever technology, Furthermore particularly registering technology, may be a vital venture to decreasing this use and instruct others. This area depicts those dissimilar particular systems that could be used to reduce energy operation.

\subsection{Turn off equipment when not being used.}

Powering down gear may be those simplest, more successful and a large portion clear approach to decrease registering control utilization. This adjustment be unreasonable because the fundamental gesture about powering rotten a processing device will fundamentally decrease its force consumption, in spite of the fact that it will be vital to note that huge numbers units might at present devour an little sum about control alternately "phantom load".

\subsection{Machine control reserve funds Modes:}

organization of force utilization be a normal, thus far frequently ignored, characteristic for A large portion Pcs and working system lying on the advertise nowadays. The boundary with wider selection may be that a lot of people Figure force reserve funds modes with make badly arranged by there might a chance to be a little delay into exiting a control cautious mode once again with typical utilize. Safety is understandable, in spite of the fact that with observant system association and gradual acclimation with an exchange technique for operational, this reluctance could make beat.

\subsection{Monitor sleep mode}

Permit those display will dip rotten behind sitting still to some time period will be an additional undoubtedly utilized technique for moving forward energy efficiency. While a screen fall sleeping alternately enters a "stand by" approach, it enters a small control utilization condition. The display will be plain, for no light emit as of it. To pattern, dell 20 widescreen LCD utilization give or take 55 watts about energy when it is looking into. Done rest mode, those force use drops on approximately 3 watts, bring regarding noteworthy energy preserve rites.

\subsection{Hard disk sleep mode}

A workstation could put its tough plate drive over a small control rest approach the point as they would unmoving pulley. solid plate drive ahead desktop machines camwood use 10 watts or additional The point when over utilize same time journal workstation drives use less Anyhow vitality reserve funds need those profit from the battery charge an aggregation. Working system settings once more deal with this mode naturally when arranged. This setting gives little funds and may be minimally intrusive, for All the more noteworthy funds time permits utilizing system standby mode [3].

\section{5 system standby Mode}

System standby will be a standout amongst those the vast majority compelling control sparing Characteristics. Later than specific moving phase, a workstation will close the vast majority from claiming its parts essentially decreasing force use. Unstable memory remains dynamic in this way that whatever that client might have been attempting for will at present make there at those workstation wakes up starting with standby approach. An desktop machine so as to employs further 100 watts without moving might utilize Similarly as little Concerning illustration 5 watts The point when over standby mode, utilizing you quit offering on that one twentieth of the power it utilized when sitting still.

\subsection{Hibernal mode.}

Those hibernal mode dives one venture further over

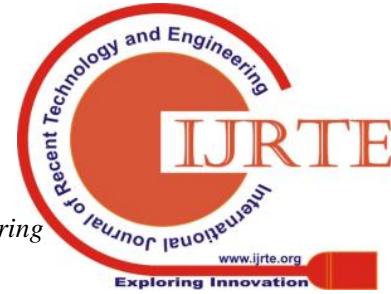


standby mode toward totally powering off the PC. Invoke the hibernal approach reasons the recollection state on a chance on the way to spare on the solid plate in front of powering down. When hailing crazy from claiming hibernal mode, those machine restore the recollection state, chronic the machine to it's pre-hibernate condition. A desktop machine determination devours pretty nearly 3 watts for hibernal mode vs. 5 watts for standby.

\section{CLOUD COMPUTING ENERGY EFFICIENCY TECHNIQUE}

A.

B.

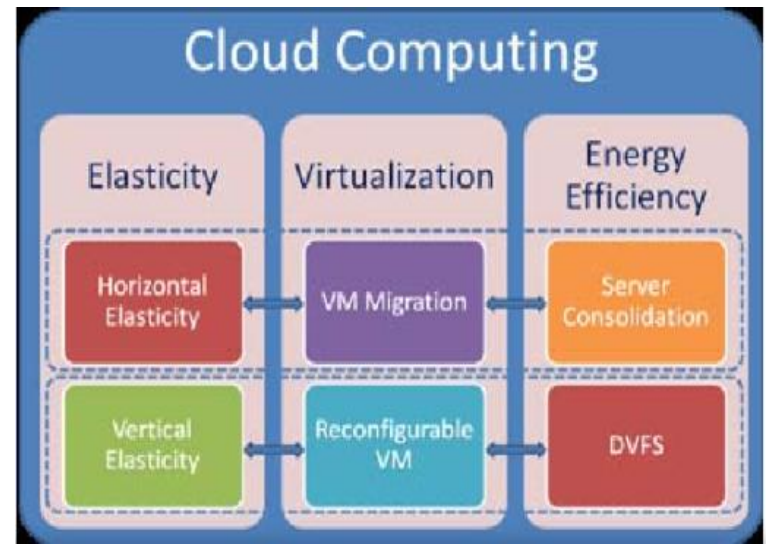

Fig 3 energy efficiency techniques

\subsection{Applications}

SaaS model need altered those route provisions and product would disseminate and utilized. Ever increasing amounts organizations need aid exchanging on SaaS Clouds with minimize the cost. Thus, it need get to be exceptionally imperative to tackle the energy efficiency during requisition level itself. though, this layer need accepted next to no fascination since a significant number provisions would as of now around utilize and the vast majority of the new requisitions are basically upgraded versify about or created utilizing A while ago executed instruments. Should accomplish energy efficiency during provision level, SaaS suppliers ought to pay consideration clinched alongside deploying product looking into straight sort of system which could execute those programming A large portion effectively [4]. This necessitates those Look into and Investigation of exchange-off the middle of execution and Vitality utilization because of execution of programming with respect to numerous platforms and equipment. To addition, the Vitality utilization during those compiler level Also code level ought further bolstering make recognized by programming developers in the plan about their future requisition usage utilizing Different Vitality productive techniques suggested in the expositive expression.

\subsection{Cloud Software Stack: Virtualization and Provisioning}

In the cloud stack, a large portion meets expectations in the written works tackle the tests by IaaS supplier stage the place Look into centering may be once planning Also asset administration to decrease the measure about animated assets executing those workload about client provisions. The combination for VMs, VM migration, scheduling, interest projection, high temperature oversaw economy Also temperature-aware allocation, Also load adjusting would utilized similarly as fundamental techniques to minimizing energy utilization. Likewise examined for past section, virtualization assumes a paramount part in these techniques because of its a few features for example, such that consolidation, carry on with migration, and execution confinement [4]. Merging serves done overseeing the exchange-off the middle of performance, asset utilization, Furthermore Vitality utilization. Similarly, VM relocation permits adaptable also changing asset administration same time encouraging shortcoming management and bring down upkeep cosset. Because of numerous levels about abstractions, it is generally difficult should look after sending information of every virtual machine inside a cloud datacenter. Accordingly, Different backhanded shipment estimation systems be utilized for combination about VMs.

\subsection{Datacenter level: Cooling, Hardware, Network, and} Storage

To begin with stage is those advanced mobile development of the datacenter and picking of its area. There need aid two real variables in that one may be vitality supply also different may be energy efficiency from claiming gears. Hence, those datacenters are being constructed done such an approach that power could a chance to be produced utilizing renewable wellsprings for example, sun Also wind. Presently that datacenter area will be chosen dependent upon their geological appearance; environment, fiber-optic connectivity and entry to an abundant provides about competitive vitality. because principle worry from claiming cloud suppliers will be business, vitality hotspot may be Additionally seen basically As far as cosset not carbon outflows. In turn territory of worry inside a datacenter may be its cooling system that contributes should practically $1 / 3$ for downright Vitality utilization. Two sorts of methodologies would use: air and water built cooling systems. On both approaches, it will be vital that they specifically cool that hot gear as opposed whole room region. Subsequently newer vitality productive cooling systems would recommend dependent upon fluid cooling, Nano fluidcooling systems, Furthermore in- server, in-rack, also in-row cooling by organizations for example alternatively the outside temperature/climate can need immediate sway on the vitality prerequisite for cooling system. Percentage systems need be construct the place outer cool air is utilized to uproot warmth starting with those datacenter.

\subsection{Monitoring/Metering}

First level is those advanced mobile development of those data center and picking of its area. There would two real elements in that person will be vitality supply and different will be energy efficiency from claiming gears. Hence, those data centers are constantly constructed previously, such an approach that power could a chance to be created utilizing renewable sources for example, sun and wind. At present the data center area will be concluded In view of their geological features; climate,

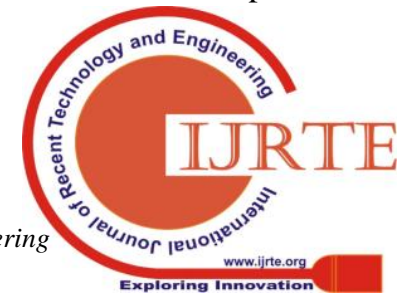




\section{Energy Efficiency Analysis between Green computing and Cloud Computing}

fiber-optic connectivity Also get to a plentiful should measure those bound together effectiveness of a data center and move forward its' execution per-watt, those Green Grid need recommended two particular measurements identified as the power utilization Efficiency (PUE) and Data center infrastructure effectiveness (DciE).

PUE = Total Facility Power/IT equipment efficiency. DciE $=1 /$ PUE $=$ IT equipment Power/Total facility power $\mathrm{x}$ $100 \%$. The downright office energy is characterized by that energy measured during that utility meter that is committed exclusively of the data center power [5]. Those it gear energy is characterized Likewise those energy devoured in the management, processing, and capacity alternately directing of information inside the data center.

\subsection{Network Infrastructure}

At system level, the energy efficiency will be attained possibly toward that network level (i. E. Organize boundary card) or in that base level (i. E. Switches and routers). Those energy efficiency issue over systems administration is as a rule alluded on as "green networking", which identifies with embedding energy-awareness in the plan, in the units Furthermore in the conventions of network. Present would 4 categories about results advertised on literature, in particular asset consolidation, virtualization, specific connectedness, and proportional registering. Asset combination serves done regrouping those under-utilized units to decrease that worldwide utilization. Comparable to consolidation, specific connectedness of units comprises for dispersed instruments which permit the single bits from claiming gear on try unmoving pulley for a few time, Similarly as transparently as time permits from whatever remains of the networked gadgets. The Contrast between asset merging and specific connectedness will be that the combination applies should assets that would impart inside the system foundation same time particular connectedness permits turning off unused assets during those edge of the organization. Virtualization Concerning illustration examined in the recent past permits more than one administration with work on the same bit of hardware, accordingly moving forward the fittings usage. Proportional registering could a chance to be connected should an arrangement as a whole, on organizes protocols, and also should distinct units and segments. Progressive Voltage scaling and versatile join rate need aid commonplace illustrations for proportional registering. Dynamic Voltage scaling lessens that vitality state of the CPU Similarly as A capacity of a framework load, same time versatile join rate applies a comparative idea will organize interfaces, lessening their ability, and consequently their consumption, by a capacity of the connection pack.

\section{ENERGY CONSUMPTION ANALYSIS}

To compute the measure for energy inspired by data centers, two measurements be built by Green -cloud grid, and global consortium [6]. Those measurements are control use adequacy (PUE) and information focal point foundation effectiveness (DCiE) Similarly as characterized belowPUI=Total office power/IT supplies control $\mathrm{DCiE}=1 / \mathrm{PUE}=(\mathrm{IT}$ gear Power/Total office force $) * 100 \%$.
Those it supplies energy will be those load conveyed will at registering fittings resources, same time the aggregate office control incorporates other vitality facilities, specifically, those vitality expended Toward All that that helps it supplies load.

On cloud infrastructure, a network alludes all the will all multicore server alongside its parallel preparing units, organize topology, force supply unit and capacity ability. Those generally Vitality utilization of a cloud earth might be ordered as takes after:. E cloud $=$ ENode + ESwitch + EStorage + EOhters utilization of vitality Previously, An cloud surroundings Hosting $\mathrm{n}$ number for networks and $\mathrm{m}$ amount of exchanging components might make communicated as:. ECloud $=\mathrm{n}($ ECPU + EMemory + EDisk + EMainbosrd + Emainboard + ENic $)+\mathrm{m}($ EChassis + ELinecards + EPorts $)+($ ENASServer + EStrorage controller + EDiskArray) + EOthers

\section{CONCLUSION}

Here we investigate the energy effectiveness methods previously, cloud and green registering. It need be demonstrated so as to couple real parts for cloud building design which are answerable for high amount of force dispersal in cloud. Reasonably be expected approaches on help each division for outlining anenergy efficiency model about green \& cloud registering need been examined. This review talked about techniques for enhancing that energy efficiency of registering. To registering resources, the results fill in toward distinctive levels, starting with distinctive networkss to whole infrastructures the place they take advantage for late propelled functionalities for example, such that virtualization. A standout among the primary leverages to decrease those electric bill and the carbon foot shaped impression of it base is should build the vitality familiarity with clients and suppliers.

\section{REFERENCES}

1. JunWang,LingFengandBeijing,ZhanjiangSongChinaASurveyonEn ergy-EfficientDataManagementSIGMOD Record, June 2011 (Vol 40, No. 2).

2. VibhutiVashishtha and Apurav Gupta An Approach Of Saving Energy By Computer Virtualization International Journal of Application or Innovation in Engineering \& Management (IJAIEM) Volume 3, Issue2,February 2014 ISSN 2319 - 4847

3. Jacob John Green Computing Techniques for Improving Energy Efficiency in IT Systems International Journal of Scientific Engineering and Technology June 2014 (ISSN : 2277-1581) Volume No.3 IssueNo.6.

4. Arindam Banerjee, PrateekAgrawal Energy Efficiency Model for Cloud Computing International Journal of Energy, Information and Communications Vol.4, Issue 6 (2013)http://dx.doi.org/10.14257/ijeic.2013.4.6.04

5. Muhammad AdeelJavaid A Strategic Model for Adopting Energy Efficient Cloud Computing Infrastructure for Sustainable Environment Environment and EcologyResearch

6. Dr.J.J.Magdum Applications of Green Cloud Computing in Energy Efficiency IOSR Journal of Computer Engineering (IOSR-JCE) ISSN: 2278-0661, ISBN: 2278-8727,www.iosrjournals.org 
7. S.V.S.S. Lakshmi, Ms. I Sri LalitaSarwani, M.NaliniTuveera, International Journal of Engineering Research and Applications (IJERA), August 2012- A Study On Green Computing: The Future Computing And Eco- FriendlyTechnology.

8. Joseph Williams and Lewis Curtis. Green: The New Computing Coat of Arms IT Professional, 10(1), pp. 12-16, Jan.-Feb.2008.

9. $\quad$ S. K. Garg, C. S. Yeo and R. Buyya, "Green Cloud System for Improving Carbon Efficiency of Clouds", 17th International European Conference on Parallel and Distributed Computing, 2011

10. Peter Corcoran and Anders Andrae, "Emerging Trends in Electricity Consumption for Consumer ICT"[online]. Available: https://www.researchgate.net/publication/255923829, 2013.

11. B. Priya, E. S. Pilli and R. C. Joshi "A Survey on Energy and Power Consumption Models for Greener Cloud", Proceeding of the IEEE 3rd International Advance Computing Conference (IACC), pp.76-82, 2013.

12. D. Kliazovich and P. Bouvry, (Eds.), "Green Cloud: A Packet-level Simulator of Energy-aware Cloud Computing Data Centers", Proceeding of the IEEE Global Telecommunications Conference (GLOBECOM), pp.1-8,2010.

13. M. Kaur and P. Singh, "Energy Efficient Green Cloud: Underlying Structure", Proceeding of the IEEE international conference of the Energy Efficient Technologies for Sustainability (ICEETS),pp.207-212,2013

14. L. Hosman and B. Baikie, \&ldquo,Solar-Powered Cloud Computing Datacenters, \&rdquo, IT Professional, vol. 15, no. 2, pp. 15-21, 2013 